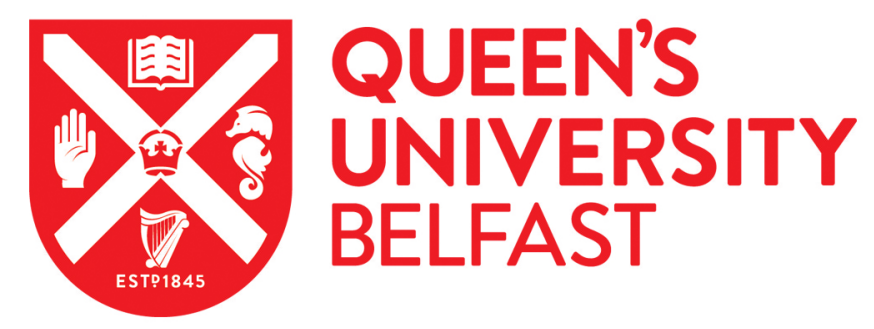

\title{
Comment on 'Contact resistance of carbon nanotubes' [Appl. Phys. Lett. 74, 2122 (1999)]
}

Delaney, P., \& Di Ventra, M. (1999). Comment on 'Contact resistance of carbon nanotubes' [Appl. Phys. Lett. 74, 2122 (1999)]. Applied Physics Letters, 75, 4028-4029. https://doi.org/10.1063/1.125527

Published in:

Applied Physics Letters

Queen's University Belfast - Research Portal:

Link to publication record in Queen's University Belfast Research Portal

\section{General rights}

Copyright for the publications made accessible via the Queen's University Belfast Research Portal is retained by the author(s) and / or other copyright owners and it is a condition of accessing these publications that users recognise and abide by the legal requirements associated with these rights.

Take down policy

The Research Portal is Queen's institutional repository that provides access to Queen's research output. Every effort has been made to ensure that content in the Research Portal does not infringe any person's rights, or applicable UK laws. If you discover content in the Research Portal that you believe breaches copyright or violates any law, please contact openaccess@qub.ac.uk. 


\title{
COMMENTS
}

\section{Comment on "Contact resistance of carbon nanotubes" [Appl. Phys. Lett. 74, 2122 (1999)]}

\author{
Paul Delaney and Massimiliano Di Ventra \\ Department of Physics and Astronomy, Vanderbilt University, Nashville, Tennessee 37235
}

(Received 25 May 1999; accepted for publication 25 October 1999)

[S0003-6951(99)03851-6]

In his recent letter, ${ }^{1}$ Tersoff has proposed that a fundamental factor that contributes to the observed high contact resistance of carbon nanotubes (NTs) on metallic surfaces is the Bloch symmetry of the wave functions of the NT. As he states, the NT wave functions at the Fermi level are formed from the graphene wave functions at graphene's $K$ points: and so, because of wave vector conservation, he finds that in the case of an armchair NT on a gold surface or embedded in gold, conduction between these NT wave functions and the gold wave functions (taken to be free-electron-like) is forbidden. This leads to the conclusion that other scattering processes are necessary to produce any conduction at all, and that the contact resistance could be decreased if more scattering was introduced, as is desirable for electronic applications.

We wish to point out that, although Tersoff's argument may apply to a sheet of graphene and gold, it does not apply to an NT, and there is indeed coupling between the NT wave functions at the Fermi level and gold wave functions, even in the absence of other scattering processes. To show this, we first go through Tersoff's argument for a sheet of graphene and gold. Following Tersoff, we assume that conduction between a NT and gold will be regulated by matrix elements of the form $M=\left\langle e^{i \mathbf{k}^{\mathrm{Au}} \cdot \mathbf{r}} \mid \Psi_{\mathbf{k}}\right\rangle$, where $\Psi_{\mathbf{k}}$ is the graphene wave function at either of the two Fermi points in its Brillouin zone (BZ) $\left(\mathbf{K}_{1},-\mathbf{K}_{1}\right.$ in Fig. 1), and the gold wave function is a plane wave, $e^{i \mathbf{k}^{\mathrm{Au}} \cdot \mathbf{r}},\left|\mathbf{k}^{\mathrm{Au}}\right| \leqslant k_{F}^{\mathrm{Au}}$. In this threedimensional integral, it is the two-dimensional part in the plane of the gold surface that yields the wave vector conservation rule $\mathbf{k}^{\mathrm{Au}}=\mathbf{k}+\mathbf{G}$, where $\mathbf{G}$ is any vector of the graphene reciprocal lattice. This comes about when we integrate $\left(1 / 4 L^{2}\right) e^{i\left(\mathbf{k}^{\mathrm{Au}}-\mathbf{k}-\mathbf{G}\right) \cdot \mathbf{r}}$ from $-L$ to $+L$ along both planar directions, and then take the limit $L \rightarrow \infty$. From Fig. 1 we see that for wave functions at, say, the $\mathbf{k}=\mathbf{K}_{1}$ point of the graphene BZ, there are no Fourier components of the graphene $\Psi_{\mathbf{K}_{1}}$ which can overlap with the projected gold Fermi sphere and hence contribute to conduction. Let us now start to change the graphene sheet into an armchair NT by using the NT reduced BZ (the rectangle in Fig. 1). For the sake of simplicity we consider the $(1,1)$ tube but our argument is valid for any NT. The Fourier coefficients at the three points $\mathbf{K}_{1}, \mathbf{K}_{3}, \mathbf{K}_{5}$ fold back to the point $T$ (Fermi point for the NT), which is two-thirds of the way towards the edge $X$ of the NT BZ. The NT wave function at $T$ is thus a linear combination of the $\mathbf{K}_{1}, \mathbf{K}_{3}, \mathbf{K}_{5}$ fourier components. Taking $a=1.42 \sqrt{3} \AA$ to be the length of the graphene realspace lattice vectors, we have

$$
\begin{aligned}
e^{i \mathbf{K}_{1} \cdot \mathbf{r}} & =e^{i(2 / 3)(2 \pi / a) \hat{\mathbf{z}} \cdot \mathbf{r}} \\
& =e^{-i(1 / 3)(2 \pi / a) \hat{\mathbf{z}} \cdot \mathbf{r}} e^{i(2 \pi / a) \hat{\mathbf{z}} \cdot \mathbf{r}} \\
& =e^{i \mathbf{k}_{T} \cdot \mathbf{r}} e^{i(2 \pi / a) \hat{\mathbf{z}} \cdot \mathbf{r}}
\end{aligned}
$$

for the Fourier component of $\Psi_{\mathbf{K}_{1}}$ at $\mathbf{K}_{1}$ and

$$
\begin{aligned}
e^{i \mathbf{K}_{3} \cdot \mathbf{r}} & =e^{i(2 / 3)(2 \pi / a)[-(1 / 2) \hat{\mathbf{z}}-(\sqrt{3} / 2) \hat{\mathbf{y}}] \cdot \mathbf{r}} \\
& =e^{i \mathbf{k}_{T} \cdot \mathbf{r}} e^{-i(1 / \sqrt{3})(2 \pi / a) \hat{\mathbf{y}} \cdot \mathbf{r}}
\end{aligned}
$$

for the Fourier component of $\Psi_{\mathbf{K}_{1}}$ at $\mathbf{K}_{3}$, and similarly for $\mathbf{K}_{5}$. Although the new $\mathbf{k}_{T}$ is now within the gold Fermi sphere (see Fig. 1) so that we can find a gold $\mathbf{k}^{\mathrm{Au}}=-(1 / 3)$ $\times(2 \pi / a) \hat{\mathbf{z}}$ that cancels the Bloch oscillation in $M$ along $z$, the contribution to $M$ is still zero for each of these three Fourier components when we integrate along both planar directions. However, when we form the NT by rolling up the graphene sheet we can no longer integrate over the whole $y$ axis, as the tube is finite in the circumferential direction. We note that the Fourier component at $\mathbf{K}_{1}$ depends only on $z$, the tube axis, and the infinite integral along $z$ would remove the $\mathbf{K}_{1}$ conduction contribution. On the other hand, the components at $\mathbf{K}_{3}$ and $\mathbf{K}_{5}$ contribute to the conduction since for a

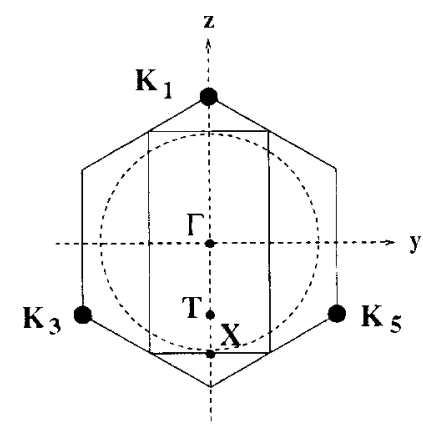

FIG. 1. Brillouin zones for graphene (hexagon) and an armchair $(1,1)$ tube (rectangle), with the tube axis along the $z$ direction, and $y$ labeling the circumferential direction. The positions of the Fourier coefficients for the graphene wave function $\Psi_{\mathbf{K}_{1}}$ are shown as large dots. The projection of the gold Fermi sphere is also shown. 
$\mathrm{NT}$, at most we can integrate over a finite region in $y$ where the NT is close to the gold; and if it is on a surface, then outside this region we must allow for the exponential decay of the NT wave function as it lifts away from the surface. So the integral involved in $M$ no longer enforces wave vector conservation, and thus the NT will carry current from gold or most other metals. The same will also be true for the small- gap $(n, m)$ tubes. We therefore conclude that the high contact resistance of NTs must be ascribed to a different mechanism.

This work was supported in part by Grant No. MDA97298-1-0007 from DARPA.

${ }^{1}$ J. Tersoff, Appl. Phys. Lett. 74, 2122 (1999). 